\title{
Differentially Coherent Code Acquisition in the Multiple Transmit/Receive Antenna Aided DS-CDMA Downlink
}

\author{
SeungHwan Won and Lajos Hanzo \\ School of ECS, Univ. of Southampton, SO17 1BJ, UK. \\ Tel: +44-23-8059 3125, Fax: +44-23-8059 4508 \\ E-mail:\{shw04r, lh\}@ecs.soton.ac.uk \\ http://www-mobile.ecs.soton.ac.uk
}

\begin{abstract}
In this contribution we investigate both differentially coherent and noncoherent code acquisition schemes in the multiple transmit/receive antenna aided DS-CDMA downlink, when communicating over uncorrelated Rayleigh channels. It is demonstrated that in contrast to our expectations, the achievable Mean Acquisition Time (MAT) degrades at low $E_{c} / I_{O}$ values, as the number of transmit antennas is increased in both differentially coherent and noncoherent code acquisition system scenarios, even though the degree of performance degradation depends upon the specific scheme considered. Ironically, our findings suggest that increasing the number of transmit antennas in a MIMO-aided CDMA system results in combining the low-energy, noise-contaminated signals of the transmit antennas, which ultimately increases the MAT by an order of magnitude, when the SINR is relatively low. Therefore our future research will be aimed at specifically designing acquisition schemes for MIMO systems.
\end{abstract}

\section{INTRODUCTION}

The application of multiple antennas in the downlink of wireless systems constitutes an attractive technique of reducing the detrimental effects of time-variant multi-path fading environments [1]. In intercell synchronous CDMA systems the mobile station's (MS) receiver must be capable of synchronising a locally generated pseudonoise (PN) code with the received multi-user signals containing the desired user's PN sequence. Substantial research efforts have been devoted to the design of code acquisition techniques [2],[3]. However, most of them have been designed for Singe-Input Single-Output (SISO) systems. There is a paucity of code acquisition techniques designed for transmit diversity aided systems [4]. Moreover, since there are no in-depth studies representing the fundamental characteristics of code acquisition schemes assisted by multiple transmit/receive antennas in the context of differentially coherent code acquisition schemes, this is the objective of the present contribution. On the other hand, there have been numerous contributions on code acquisition techniques designed for receive diversity aided systems [5],[6]. Similarly to noncoherent code acquisition schemes [2],[3], differentially coherent code acquisition [7] does not require any prior information on the carrier phase. An additional benefit of employing a differentially coherent code acquisition scheme is that it is capable of providing a better performance than using a noncoherent one [2],

The financial support of the Ministry of Information and Communication(MIC), Republic of Korea and the European Union under the auspices of the Phoenix and Newcom projects and that of the EPSRC is gratefully acknowledged.
[3], [7], [8]. Here we adopted the Full-Period Correlation (FPC) based scheme of [7],[8] for the sake of analysing the characteristics of serial search aided differentially coherent code acquisition in the multiple transmit/receive antenna aided DS-CDMA downlink. Against this background, in this treatise we investigate both serial search based differentially coherent and noncoherent code acquisition schemes designed for multiple transmit/receive antenna aided systems. More explicitly, we quantify both the correct detection probability as well as the false alarm probability as a function of both the Signalto-Interference plus Noise Ratio (SINR) per chip $\left(E_{c} / I_{o}\right)$ and that of the number of transmit/receive antennas. Furthermore, we characterise the MAT performance versus the detection threshold value, parameterised by both the $E_{c} / I_{o}$ values and the number of transmit/receive antennas. This paper is organised as follows. The MAT expressions are introduced and interpreted in Section II, while Section III describes the system investigated, followed by the correct detection and false alarm probability analysis of both differentially coherent and noncoherent code acquisition schemes in the context of uncorrelated Rayleigh channels. In Section IV, our numerical Mean Acquisition Time (MAT) results are discussed and our conclusions are offered in Section V.

\section{MEAN ACQUISITION TIME ANALYSIS}

In [9],[10], explicit MAT formulae were provided for a singleantenna aided serial search based code acquisition system. There is no difference between a single-antenna aided scheme and a multipleantenna assisted one in terms of analysing the MAT, except for deriving the correct detection and the false alarm probability based upon the multiple transmit/receive antennas. We will commence our discourse by comparing the MAT performance of differential FPC based code acquisition to that of the corresponding noncoherent one using Single Dwell Serial Search (SDSS) [7], because the correlation operation of the FPC scheme is performed over a full code period [7]. We assume that in each chip duration $T_{c}, l$ number of correct timing hypotheses are tested, which are spaced by $T_{c} / l$, and hence the total uncertainty region is increased by a factor of $l$. All the resultant $(\nu-2 l)$ states that may lead to a false alarm are expected to increase the MAT according to the corresponding penalty time. The $2 l$ legitimate locking states within a lag of one chip duration of the correct timing instant are taken into account in the MAT 
analysis. Combining all these $2 l$ legitimate locking states into the correct detection transfer function encompassing all branches of the relevant state diagram [9] leads to the correct detection transfer function expressed as

$$
H_{D}(z)=\sum_{j=1}^{2 l} P_{D j} z \prod_{i=1}^{j-1}\left[\left(1-P_{D i}\right) z\right],
$$

where $P_{D j}$ represents the correct detection probability associated with the $j^{\text {th }}$ correct detection, following $(j-1)$ trials each resulting in a miss and where each probability of a miss in the corresponding total miss transfer function is given by $\left(1-P_{D i}\right)$. Furthermore, $z$ indicates the unit-delay operator and the exponent of $z$ represents the time delay, while $H_{0}(z)$ denotes the absence of the desired user's signal at the output of the acquisition scheme, which is expressed as

$$
H_{0}(z)=\left(1-P_{F}\right) z+P_{F} z^{K+1},
$$

where $P_{F}$ represents the false alarm probability [9] and $K$ is the false locking penalty factor [9]. Finally, $H_{M}(z)$ represents the overall miss probability of a search run carryed out across the entire uncertainty region, which may be formulated as the product of the individual miss probabilities, since these may be considered independent events, yielding

$$
H_{M}(z)=z^{2 l} \prod_{j=1}^{2 l}\left(1-P_{D j}\right) .
$$

It may be shown that the generalised expression derived for computing the MAT of the serial search based code acquisition scheme considered is given by [9]:

$$
\begin{array}{r}
E\left[T_{A C Q}\right]=\frac{1}{H_{D}(1)}\left[H_{D}{ }^{\prime}(1)+H_{M}{ }^{\prime}(1)+\right. \\
\left.\left\{(\nu-2 l)\left[1-\frac{H_{D}(1)}{2}\right]+\frac{1}{2} H_{D}(1)\right\} H_{0}{ }^{\prime}(1)\right] \cdot \tau_{D},
\end{array}
$$

where $\left.H_{x}^{\prime}(z)\right|_{x=D, M \text { or } 0}$ is the derivative of $\left.H_{x}(z)\right|_{x=D, M \text { or } 0}$ and $\tau_{D}$ the dwell time [9].

\section{THEORETICAL ANALYSIS OF BOTH DIFFERENTIALLY COHERENT AND NONCOHERENT CODE ACQUISITION}

The received signal of the MIMO-aided differentially coherent DSCDMA downlink may be expressed as

$$
\begin{array}{r}
r(t)=\sum_{m=1}^{P} \sum_{n=1}^{R}\left[\alpha_{m, n} \sqrt{\frac{E_{c}}{P T_{c}}} c\left(t+d T_{c}\right) w_{m}\left(t+d T_{c}\right)\right. \\
\left.\cdot \exp \left(2 \pi f t+\phi_{m, n}\right)+I_{k(m, n)}(t)\right],
\end{array}
$$

where $m=1, \ldots, P$ is the number of transmit antennas, $n=1, \ldots, R$ is the number of receive antennas, $\alpha_{m, n}$ represents the envelope of the $(m, n)^{t h}$ path signal obeying the Rayleigh distribution, $E_{c}$ denotes the pilot signal energy per PN code chip, $c(t)$ denotes a unique userspecific PN sequence, $d$ is the code phase offset with respect to the phase of the local code, $T_{c}$ is the chip duration, $w_{m}(t)$ identifies the specific Walsh code assigned to the $m^{\text {th }}$ transmit antenna, $f$ is the carrier frequency and finally, $\phi$ is the carrier phase of a specific user's modulator. Furthermore, $I_{k(m, n)}(t)$ is the complex-valued additive white Gaussian noise (AWGN) having a double-sided power spectral density of $I_{0}$ at the $(m, n)^{t h}$ path. Here the total allocated power is equally shared by the $P$ transmit antennas. Fig. 1 depicts the block diagram of the differentially coherent receiver designed for our code acquisition scheme using multiple transmit/receive antennas. In this figure, instead of squaring the accumulated energy as suggested by the procedures outlined in [9], the channel's output samples summed over a full spreading code period are multiplied by the conjugate of the $N$-chip-delayed samples [8].

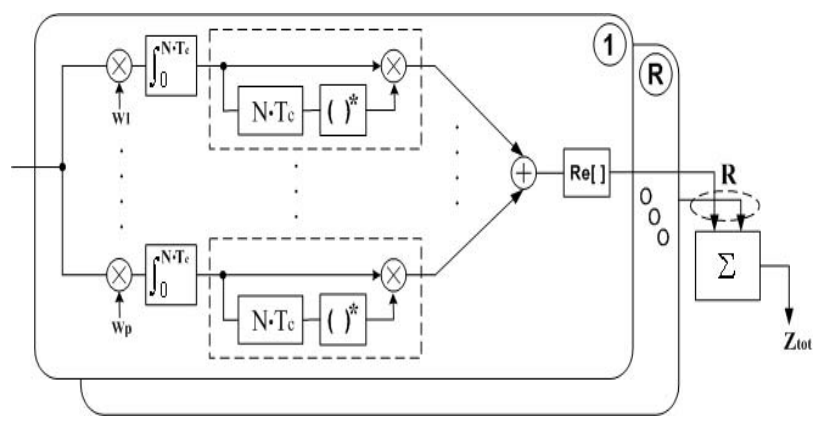

Fig. 1. Receiver structure of a differentially coherent code acquisition system using $R$ receive antennas.

A. Correct Detection and False Alarm Probability of the Differentially Coherent Code Acquisition Scheme

A decision variable is generated by accumulating $(P \cdot R)$ number of independently faded received signals observed over a time interval for the sake of improving the correct detection probability in the mobile channel imposing both fading and poor SINR conditions, where $P$ and $R$ are the number of transmit and receive antennas, respectively. Owing to space limitations, we omitted formulating the final decision variable, which is readily derived from the procedures proposed in [8] in the context of the receiver structure of Fig.1. The final differentially coherent $(\mathrm{dc})$ decision variable may be written as [8]

$$
\begin{aligned}
& Z_{k}^{d c}=\sum_{m=1}^{P} \sum_{n=1}^{R}\left[\left(\sqrt{\frac{4 E_{c}}{N I_{0} P}} \cdot S_{k(m, n)}+W_{1, k(m, n)}\right)^{2}\right. \\
& \left.+W_{3, k(m, n)}^{2}\right]-\sum_{m=1}^{P} \sum_{n=1}^{R}\left[W_{2, k(m, n)}^{2}+W_{4, k(m, n)}^{2}\right],
\end{aligned}
$$

where $k$ denotes the $k^{\text {th }}$ chip's sampling instant, $S_{k(m, n)}$ is assumed to be deterministic [8] and the definition of $W_{1, k(m, n)}, W_{2, k(m, n)}$, $W_{3, k(m, n)}$ and $W_{4, k(m, n)}$ is also the same as in [8], which are mutually independent Gaussian random variables having zero means and unit variances [8]. Let us now introduce a shorthand for the first and second terms of $\mathrm{Eq}(6)$ as follows:

$$
\begin{array}{r}
X_{k}=\sum_{m=1}^{P} \sum_{n=1}^{R}\left[\left(\sqrt{\frac{4 E_{c}}{N I_{0} P}} \cdot S_{k(m, n)}+W_{1, k(m, n)}\right)^{2}\right. \\
\left.+W_{3, k(m, n)}^{2}\right]
\end{array}
$$


and

$$
Y_{k}=\sum_{m=1}^{P} \sum_{n=1}^{R}\left[W_{2, k(m, n)}^{2}+W_{4, k(m, n)}^{2}\right] .
$$

Then the final decision variable of $\mathrm{Eq}(6)$ is obtained as $Z_{k}^{d c}=$ $X_{k}-Y_{k}=\sum_{m=1}^{P} \sum_{n=1}^{R} X_{k(m, n)}-\sum_{m=1}^{P} \sum_{n=1}^{R} Y_{k(m, n)}$, where $X_{k}$ obeys a noncentral chi-square PDF with $(2 P \cdot R)$ degrees of freedom and its noncentrality parameter $\lambda_{x}$ is either $\frac{4 N}{P}\left(\frac{E_{c}}{I_{0}}\right)^{\prime}$, when the desired signal is deemed to be present $(x=1)$ or $\frac{4}{N P}\left(\frac{E_{c}}{I_{0}}\right)^{\prime}$, when it is deemed to be absent $(x=0)$ [8]. The effects of both timing errors and frequency mismatches are encapsulated by the definition of $\left(E_{c} / I_{0}\right)^{\prime}$, in the spirit of [9], while $Y_{k}$ is centrally chi-square distributed with $(2 P \cdot R)$ degrees of freedom. Therefore, the PDF of $X_{k}$ and $Y_{k}$ can be shown to be [11]

$$
\begin{array}{r}
f_{X_{k}}\left(z \mid H_{x}\right)=\frac{1}{2}\left(\frac{z}{\lambda_{x}}\right)^{\frac{(P R-1)}{2}} \\
\cdot \exp \left[-\frac{\left(z+\lambda_{x}\right)}{2}\right] \\
\cdot \mathcal{I}_{(P R-1)}\left(\sqrt{z \cdot \lambda_{x}}\right)
\end{array}
$$

and

$$
f_{Y_{k}}\left(z \mid H_{x}\right)=\frac{1}{2^{P R} \cdot \Gamma(P R)} \cdot z^{(P R-1)} \cdot \exp \left[-\frac{z}{2}\right],
$$

respectively, where $z \geq 0, x=0$ or $1, \Gamma(\cdot)$ is the Gamma function and $\mathcal{I}_{(P R-1)}(\cdot)$ is the $(P \cdot R-1)^{s t}$-order modified Bessel function. Our aim is now that of expressing the PDF of a desired user's signal at the output of the acquisition scheme conditioned on the presence of the desired signal in $f_{X_{k}}\left(z \mid H_{x}\right)$ derived for transmission over an uncorrelated Rayleigh channel. Hence first the PDF $f_{X_{k(m, n)}}\left(z \mid H_{x}, \beta\right)$ corresponding to a specific SINR $\beta$ conditioned on the hypothesis of the desired signal being transmitted over an AWGN channel having this specific SINR is weighted by the probability of occurrence $f(\beta)$ of encountering the SINR $\beta$, as quantified by the PDF. The resultant product is then averaged over its legitimate range of $-\infty \sim \infty$, yielding:

$$
\begin{aligned}
& f_{X_{k(m, n)}}\left(z \mid H_{x}\right)=\int_{-\infty}^{\infty} f(\beta) \cdot f_{X_{k(m, n)}}\left(z \mid H_{x}, \beta\right) d \beta \\
& =\int_{0}^{\infty}\left(\frac{e^{-\beta / \sigma^{2}}}{\sigma^{2}}\right) \cdot \frac{\exp \left[-\left(z+\beta \lambda_{x}\right) / 2\right]}{2} \\
& \cdot \mathcal{I}_{0}\left(\frac{2 \sqrt{\beta \lambda_{x} z}}{2}\right) d \beta \\
& =\frac{\exp \left[-z /\left(2+\lambda_{x} \sigma^{2}\right)\right]}{\left(2+\lambda_{x} \sigma^{2}\right)} \\
& \equiv \frac{\exp \left[-z /\left(2+\overline{\lambda_{x}}\right)\right]}{\left(2+\overline{\lambda_{x}}\right)},
\end{aligned}
$$

where the corresponding noncentrality parameter of $\overline{\lambda_{x}} \equiv \lambda_{x} \sigma^{2}$ is either $\frac{4 N}{P}\left(\frac{\overline{E_{c}}}{I_{0}}\right)^{\prime}$ when the desired signal is deemed to be present $(x=1)$ or $\frac{4}{N P}\left(\overline{E_{c}}\right)^{\prime}$ when it is deemed to be absent $(x=0)$. For the sake of notational convenience we also define a new biased noncentrality parameter $\mu_{x}=\left(2+\overline{\lambda_{x}}\right)$. Further details on the related calculations are found in [8] and [9]. Finally, we arrive at the PDF of $X_{k}(m, n)$ conditioned on the presence of the desired signal in the form of:

$$
f_{X_{k(m, n)}}\left(z \mid H_{x}\right)=\frac{1}{\mu_{x}} e^{-z / \mu_{x}} .
$$

By contrast, the PDF of $f_{Y_{k(m, n)}}\left(z \mid H_{x}\right)$ may be readily derived from (10), yielding

$$
f_{Y_{k(m, n)}}\left(z \mid H_{x}\right)=\frac{1}{2} e^{-z / 2} .
$$

Since the decision variables, $X_{k}$ and $Y_{k}$ are constituted by the sum of $(P \cdot R)$ number of independent variables $\left(X_{k}=\sum_{m=1}^{P} \sum_{n=1}^{R} X_{k(m, n)}\right.$ and $\left.Y_{k}=\sum_{m=1}^{P} \sum_{n=1}^{R} Y_{k(m, n)}\right)$, each of which has a PDF given by (15) or (16), we can determine the Laplace transform of each by raising them to the $(P \cdot R)^{t h}$ power and then carrying out the inverse transform for the sake of generating the desired PDF [9], leading to:

$$
\begin{aligned}
f_{X_{k}}\left(z \mid H_{x}\right) & =\frac{z^{(P R-1)} e^{-z / \mu_{x}}}{\Gamma(P R) \cdot \mu_{x}^{P R}}, \\
f_{Y_{k}}\left(z \mid H_{x}\right) & =\frac{z^{(P R-1)} e^{-z / 2}}{\Gamma(P R) \cdot 2^{P R}} .
\end{aligned}
$$

Then, the PDF of $Z_{k}^{d c}=X_{k}-Y_{k}$ can be computed by straightforward convolution of the PDFs of both $X_{k}$ and $Y_{k}$, which results in the PDF of the difference between two independent Gamma variables. To elaborate a little further, we have $X_{t o t} \sim g\left(P \cdot R, \mu_{x}\right)$ and $Y_{t o t} \sim g(P \cdot R, 2)$, where this shorthand indicates that both $X_{t o t}$ and $Y_{\text {tot }}$ follow a Gamma distribution having the shape parameter of $(P \cdot R)$ and the scale parameter of either $\mu_{x}$ or 2 , respectively, as outlined in [12]. The convolution of the PDFs $f_{X_{k}}$ and $f_{Y_{k}}$ derived for the sake of calculating the PDF of $Z_{k}^{d c}$ conditioned on the desired signal being present or absent is formulated as [12]:

$$
\begin{aligned}
f_{Z_{k}^{d c}}\left(z \mid H_{x}\right) & =\int_{-\infty}^{\infty} f_{X_{k}}(\xi) \cdot f_{Y_{k}}(\xi-z) d \xi \\
= & \left(\frac{\left(1-c^{2}\right)^{a+\frac{1}{2}} \cdot|z|^{a}}{\sqrt{\pi} \cdot 2^{a} \cdot b^{a+1} \cdot \Gamma\left(a+\frac{1}{2}\right)}\right) \\
& \cdot \exp \left(-\frac{c}{b} z\right) \cdot K_{a}\left(\frac{|z|}{b}\right), z \neq 0,
\end{aligned}
$$

where $K_{a}(\cdot)$ is the modified Bessel function of the second kind and of order $a$. We note furthermore that $K_{a}(\cdot)$ is undefined, when the argument is equal to zero. However, this fact has a negligible impact on calculating the probability of correct detection and false alarm. Further details on the associated statements are detailed in [12]. Let us now define the following three parameters, namely $a=P \cdot R-0.5$, $b=\left(4 \mu_{x}\right) /\left(\mu_{x}+2\right)$ and $c=-\left(\mu_{x}-2\right) /\left(\mu_{x}+2\right)$, which allow us to express the probability of correct detection or false alarm according to $x=1$ or 0 , respectively, as follows [12]:

$$
\begin{aligned}
\left.P^{d c}\right|_{x=1 \text { or } 0}= & \int_{\theta}^{\infty}\left(\frac{\left(1-c^{2}\right)^{a+\frac{1}{2}} \cdot|z|^{a}}{\sqrt{\pi} \cdot 2^{a} \cdot b^{a+1} \cdot \Gamma\left(a+\frac{1}{2}\right)}\right) \\
& \cdot \exp \left(-\frac{c}{b} z\right) \cdot K_{a}\left(\frac{|z|}{b}\right) d z, \theta \neq 0,
\end{aligned}
$$

where $\theta$ is a threshold value. 
B. Correct Detection and False Alarm Probability of the Noncoherent Code Acquisition Scheme

For the sake of comparison, the noncoherent counterpart of the previously described differentially coherent scheme is characterised here, where the final decision variable is given by [8]

$$
Z_{k}^{n c}=\sum_{m=1}^{P} \sum_{n=1}^{R}\left\|\frac{1}{\sqrt{2}} \cdot\left(\sqrt{\frac{4 E_{c}}{N I_{0} P}} \cdot S_{k(m, n)}+I_{k(m, n)}\right)\right\|^{2}
$$

where $\|\cdot\|^{2}$ represents the squared envelope of the complex-valued argument and the factor of $1 / \sqrt{2}$ is employed to normalise the noise variance. Accordingly, the noncoherent (nc) decision variable $Z_{k}^{n c}$ obeys a noncentral chi-square PDF with $(2 P \cdot R)$ degrees of freedom and its noncentrality parameter $\lambda_{x}$ is either $\frac{2 N}{P}\left(\frac{E_{c}}{I_{0}}\right)^{\prime}$ for the hypothesis of the desired signal being present $(x=1)$ or $\frac{2}{N P}\left(\frac{E_{c}}{I_{0}}\right)^{\prime}$ for it being absent $(x=0)$ [8], where the PDF is given by [11]

$$
\begin{array}{r}
f_{Z_{k}^{n c}}\left(z \mid H_{x}\right)=\frac{1}{2}\left(\frac{z}{\lambda_{x}}\right)^{\frac{(P R-1)}{2}} \cdot \exp \left[-\frac{\left(z+\lambda_{x}\right)}{2}\right] \\
\cdot \mathcal{I}_{(P R-1)}\left(\sqrt{z \cdot \lambda_{x}}\right),
\end{array}
$$

and where $z \geq 0, x=0$ or 1 . Given the $\operatorname{PDF} f_{Z_{k}^{n c}}\left(z \mid H_{x}\right)$ conditioned on the presence of the desired signal, which was derived for transmission over an uncorrelated Rayleigh channel, and following a similar procedures to that outlined in Section A, finally, the probability of correct detection or false alarm corresponding to $x=1$ or 0 , respectively is obtained as

$$
\left.P^{n c}\right|_{x=1 \text { or } 0}=\exp \left(-\frac{\theta}{\mu_{x}}\right) \cdot \sum_{k=0}^{P R-1} \frac{\left(\theta / \mu_{x}\right)^{k}}{k !}
$$

where $\mu_{x}$ is again set to be $\left(2+\overline{\lambda_{x}}\right)$ and $\overline{\lambda_{x}}$ is either $\frac{2 N}{P}\left(\overline{\frac{E_{c}}{I_{0}}}\right)$ for the hypothesis of the desired signal being present $(x=1)$ or $\frac{2}{N P}\left(\overline{E_{c}}\right)^{\prime}$ for it being absent $(x=0)$.

\begin{tabular}{|c|c|c|}
\hline \multicolumn{2}{|l|}{ Bandwidth } & $1.2288 \mathrm{MHz}$ \\
\hline \multicolumn{2}{|c|}{ Carrier frequency } & $1.9 \mathrm{GHz}$ \\
\hline \multicolumn{2}{|c|}{ Spreading factor } & 128 \\
\hline \multirow{2}{*}{ Diversity: } & Transmit & $1,2,4$ \\
\hline & Receive & $1,2,4$ \\
\hline \multicolumn{2}{|c|}{ Frequency mismatch } & $1000 \mathrm{~Hz}$ \\
\hline \multicolumn{2}{|c|}{ Mobile speed } & $160 \mathrm{~km} / \mathrm{h}$ \\
\hline \multicolumn{2}{|c|}{ Coherent integration interval } & 256chips \\
\hline \multicolumn{2}{|c|}{ Total uncertainty region } & 512 \\
\hline \multicolumn{2}{|c|}{ False locking penalty factor } & 1000 \\
\hline
\end{tabular}

\section{NUMERICAL SYSTEM PERFORMANCE RESULTS}

TABLE I

SYSTEM PARAMETERS

In this section we will characterise the MAT performance of multiple transmit/receive antenna aided DS-CDMA systems. The associated system parameters are summarised in Table 1. In Table 2 we outlined the maximum SINR degradation imposed by both the Doppler shift and the frequency mismatch between the transmitter
TABLE II

MAXIMUM SINR DEGRADATION INFLICTED BY BOTH THE DOPPLER SHIFT AND A $1000 \mathrm{HZ}$ FREQUENCY MISMATCH IN CONJUNCTION WITH THE COHERENT INTEGRATION INTERVAL OF N CHIP DURATIONS AT A CARRIER FREQUENCY OF $1.9 \mathrm{GHZ}$

\begin{tabular}{|l|l|l|l|l|l|}
\hline $\mathrm{N}$ (Chips) & 64 & 128 & 256 & 384 & 512 \\
\hline Degradation $(\mathrm{dB})$ & 0.061 & 0.2449 & 0.9969 & 2.3144 & 4.3213 \\
\hline
\end{tabular}

and receiver in conjunction with a coherent integration interval of $N$ chip durations. The length of the PN sequence in our system was assumed to be $256 T_{c}$, where the chip-duration is $T_{c}=1 / 1.2288 \mu \mathrm{s}$. It was found to be sufficient to integrate the detector output seen in Fig. 1 over $N=256$ chips, which is equivalent to two 128-chip directsequence modulated symbols used for coherent accumulation due to the adoption of the above-mentioned FPC scheme of [8]. This value was calculated by using the appropriate equations of [9] provided for determining the performance degradation owing to both the Doppler shift and the frequency mismatch. The spreading factor of the Walsh code to be acquired was selected to be 128 . The frequency mismatch was assumed to be $1000 \mathrm{~Hz}$ [9], while the carrier frequency was $1.9 \mathrm{GHz}$. As a worst-case mobile speed, it is reasonable to postulate $160 \mathrm{~km} / \mathrm{h}$. We also assumed that the sampling inaccuracy caused by having a finite, rather than infinitesimally low, search step size of $\Delta=1 / 2 T_{c}$ was $-0.91 \mathrm{~dB}$, which is a typical value for the search step size [9]. The total uncertainty region was assumed to entail 512 hypotheses. Finally, in the spirit of [13], the false locking penalty factor was assumed to be 1000 .

Fig.2 illustrates the achievable MAT versus the detection threshold value for the differentially coherent code acquisition system as a function of both the number of transmit antennas for $P=1,2$ as well as 4 and that of the SINR per chip denoted as $E_{c} / I_{o}$. Observe in Fig. 2 that surprisingly, as the number of transmit antennas is decreased, despite the potentially reduced transmit diversity gain, we experience an improved MAT performance at $E_{c} / I_{o}=-16 \mathrm{~dB}$. However, observe in Fig. 2 that in the case of $E_{c} / I_{o}=-13$ and $10 \mathrm{~dB}$, the performance degradation remains relatively low, as the transmit diversity order is increased from $P=1$ to 4 . For comparison, Fig. 3 characterises the achievable MAT versus the detection threshold value for the noncoherent code acquisition arrangement as a function of the number of transmit antennas for $P=1,2$ as well as 4 and that of the SINR per chip. Similarly to the conclusions of Fig.2, as the number of transmit antennas is decreased, all the curves seen in Fig.3 illustrate an improved MAT peformance. However, unlike in the case of the differentially coherent scheme of Fig.2, the MAT performance degradation becomes less pronounced for higher $E_{c} / I_{o}$ values such as $E_{c} / I_{o}=-10 \mathrm{~dB}$, as the transmit diversity order is increased from $P=1$ to 4 . This is because the differentially coherent scheme characterised in Fig. 2 has a performance gain of just under $3 \mathrm{~dB}$ in comparison to the noncoherent one. The MAT performance results of Fig.2 confirm the expected trends. Moreover, 
this explicitly indicates that the differentially coherent scheme also degrades the achievable MAT performance as a consequence of employing multiple transmit antennas for the sake of attaining a transmit diversity gain. However, the MAT peformance degradation imposed is less severe than that of noncoherent counterpart. Fig.4 and Fig. 5 characterise the achievable MAT versus detection threshold performance, parameterised by both the number of transmit antennas for $P=1,2$ as well as 4 in conjunction with $R=2$ receive antennas and by the $E_{c} / I_{o}$ ratio for both the differentially coherent code acquisition scheme (Fig.4) and for its noncoherent counterpart (Fig.5). The achievable MAT versus detection threshold performance recorded for exactly the same parameters as in Fig.4 and Fig.5 but using $R=4$ receive antennas is characterised in Fig.6 and Fig.7, respectively. When having $R=2$ and $R=4$ receive antennas, similar trends may be observed for both differentially coherent code acquisition schemes as portrayed in Fig.4 and Fig.6, respectively. By contrast, as seen in Fig.5 and Fig.7 for the same scenario of $R=2$ and $R=4$ receive antennas, respectively, the noncoherent code acquisition scheme exhibited a more drastic MAT performance degradation at relatively low $E_{c} / I_{o}$ values such as $-16 \mathrm{~dB}$, compared to differentially coherent code acquisition, especially, as the number of transmit antennas was increased. To elaborate a little further, in case of employing both multiple transmit and receive antennas, similar trends are still observable, although using two or four receive antennas has the potential of mitigating the associated acquisition performance degradation imposed by the low per-branch $E_{c} / I_{o}$ values associated with the employment of multiple transmitters. To interpret the above results a little further, a low level of perbranch received signal strength would lead to a low inital acquisition performance, despite achieving a high transmit diversity gain. In other words, a high transmit diversity order effectively results in an initial acquisition performance loss, as a consequence of the insufficiently high signal strength per transmit antenna branch.

\section{CONCLUSION}

In this paper, we analysed the multiple antenna aided transmit/receive diversity effects on the initial acquisition performance of both differentially coherent and noncoherent code acquisition schemes in the inter-cell synchronous CDMA downlink. The probabilities of correct detection and false alarm have been derived analytically and numerical results were provided in terms of the MAT performance. Ironically, our findings suggest that increasing the number of transmit antennas in a MIMO-aided CDMA system results in combining the low-energy, noise-contaminated signals of the transmit antennas, which ultimately increases the MAT by an order of magnitude, when the SINR is relatively low. However, it is extremely undesirable to degrade the achievable initial acquisition performance, when the perfectly synchronised system is capable of attaining its target bit error rate performance at reduced SINR values, as a benefit of employing multiple transmit antennas. Therefore our future research will be aimed at specifically designing acquisition schemes for MIMO systems.

\section{REFERENCES}

[1] D. Gesbert, M. Shafi, D.S. Shiu, P.J. Smith, and A. Naguib, From Theory to Practice: An Overview of MIMO Space-Time Coded Wireless Systems, IEEE Journal on Selected Areas in Communications, vol. 21, NO.3, Issue 3, 2003, pp281-302.

[2] B-G Lee, B-H Kim, Scrambling Techniques For CDMA Communications, Chapter 2 and 3, Kluwer Academic Publishers, 2001.

[3] L. Hanzo, L-L Yang, E-L Kuan, K. Yen, Single- and MultiCarrier DS-CDMA, IV Multi-Carrier CDMA, Chapter 21, Initial Synchronization of DS-CDMA and MC-CDMA Systems, Wiley, 2003.

[4] S.H. Won and Y.J. Kim, Performance Analysis of Multi-path Searcher for Mobile Station in W-CDMA System Employing Transmit Diversity, Electronics Letters, vol. 39, Issue 1, 2003, pp137-139.

[5] R.R. Rick and L.B. Milstein, Parallel Acquisition Schemes Using Antenna Arrays for Antenna Diversity, IEEE Transaction on Communications, vol. 45, NO. 8, Issue 8, 1997, pp903-905.

[6] S. Kim, Acquisition Performance of CDMA Systems with Multiple Antennas, IEEE Transactions on Vehicular Technology, vol. 53, NO. 5, Issue 1, 2004, pp1341-1353.

[7] M.H. Zarrabizadeh, E.S. Sousa, A differentially coherent PN code acquisition receiver for CDMA systems, IEEE Transactions on Communications, vol. 45, NO.11, Issue 11, 1997, pp1456-1465.

[8] J-C Lin, Differentially coherent PN code acquisition with full-period correlation in chip-synchronous DS/SS receivers, IEEE Transactions on Communications, vol. 50, NO.5, Issue 5, 2002, pp698-702.

[9] A.J. Viterbi, CDMA: Principles of Spread Spectrum Communication, Chapter 3, Addison-Wesley, 1995.

[10] L-L Yang, L. Hanzo, Serial Acquisition of DS-CDMA Signals in Multipath Fading Mobile Channels, IEEE Transactions on Vehicular Technology, vol. 50, NO.2, Issue 2, 2001, pp617-628.

[11] J.G. Proakis, Digital Communications, 3rd ed. Chapter 2, McGrawHill, 1995

[12] H. Holm, M-S. Alouini, Sum and Difference of Two Squared Correlated Nakagami Variates in Connection with the McKay Distribution, IEEE Transactions on Communications, vol. 52, NO.8, Issue 8, 2004, pp1367-1376.

[13] H.R. Park, Performance Analysis of a Double-Dwell Serial Search Technique for Cellular CDMA Networks in the Case of Multiple Pilot Signals, IEEE Transactions on Vehicular Technology, vol. 48, NO.6, Issue 6, 1999, pp1819-1830. 


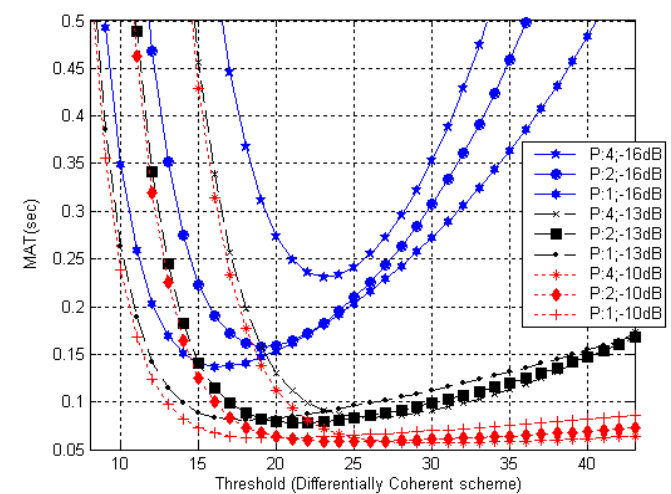

Fig. 2. MAT versus the detection threshold value of the differentially coherent code acquisition system for $P=1,2$ as well as 4 transmit antennas in conjunction with $R=1$ receive antenna for transmission over uncorrelated Rayleigh channels.

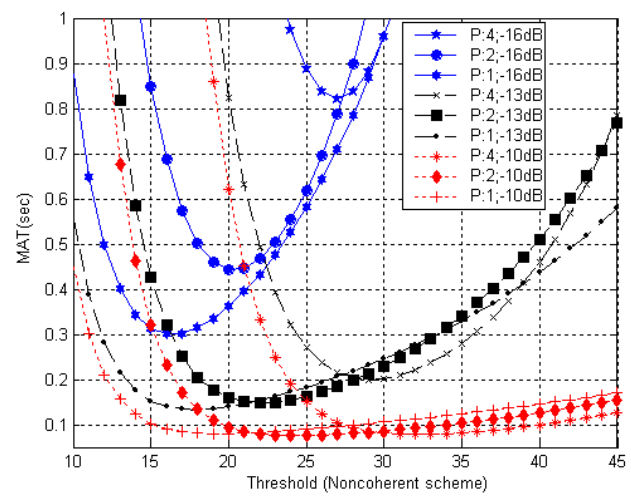

Fig. 3. MAT versus the detection threshold value of the noncoherent code acquisition system for $P=1,2$ as well as 4 transmit antennas in conjunction with $R=1$ receive antenna for transmission over uncorrelated Rayleigh channels.

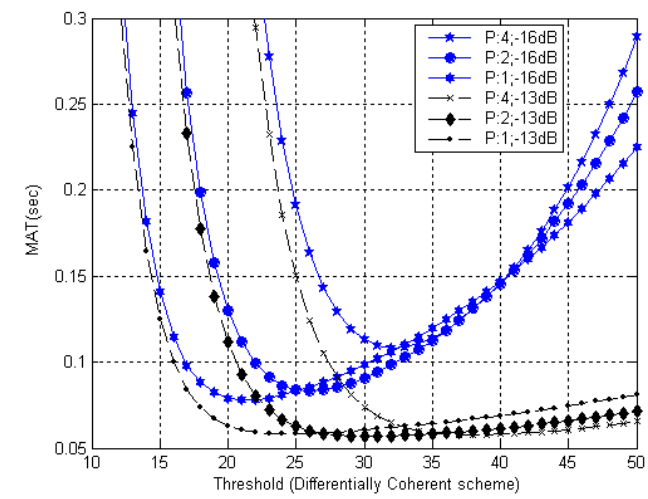

Fig. 4. MAT versus the detection threshold value of the differentially coherent code acquisition system for $P=1,2$ as well as 4 transmit antennas in conjunction with $R=2$ receive antenna for transmission over uncorrelated Rayleigh channels.

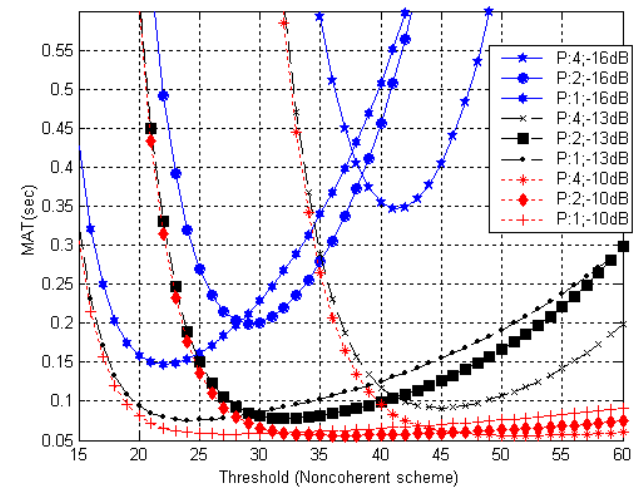

Fig. 5. MAT versus the detection threshold value of the noncoherent code acquisition system for $P=1,2$ as well as 4 transmit antennas in conjunction with $R=2$ receive antenna for transmission over uncorrelated Rayleigh channels.

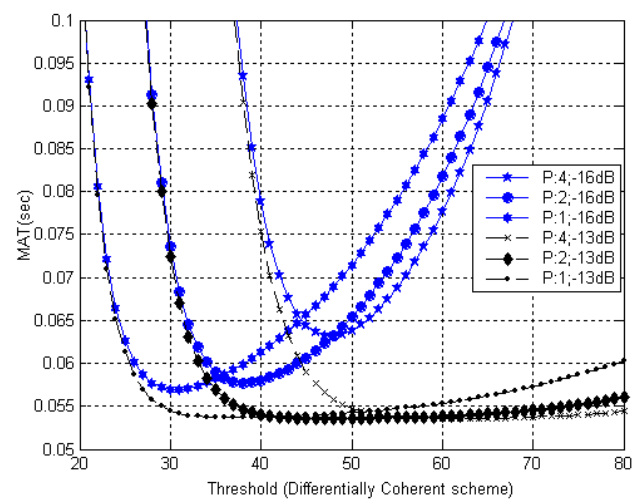

Fig. 6. MAT versus the detection threshold value of the differentially coherent code acquisition system for $P=1,2$ as well as 4 transmit antennas in conjunction with $R=4$ receive antenna for transmission over uncorrelated Rayleigh channels.

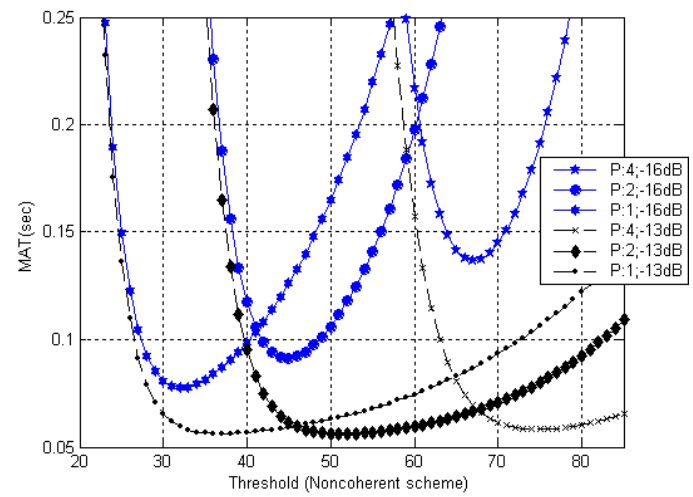

Fig. 7. MAT versus the detection threshold value of the noncoherent code acquisition system for $P=1,2$ as well as 4 transmit antennas in conjunction with $R=4$ receive antenna for transmission over uncorrelated Rayleigh channels. 